\title{
Sinonasal outcomes following endoscopic anterior skull base surgery with nasoseptal flap reconstruction: a prospective study
}

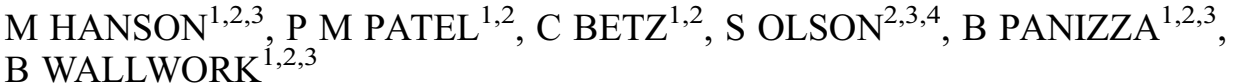

Departments of ${ }^{1}$ Otolaryngology, Head and Neck Surgery, and ${ }^{4}$ Neurosurgery, Princess Alexandra Hospital, Woolloongabba, ${ }^{2}$ School of Medicine, University of Queensland, Woolloongabba, and ${ }^{3}$ Greenslopes Private Hospital, Queensland, Australia

\begin{abstract}
Objective: To assess nasal morbidity resulting from nasoseptal flap use in the repair of skull base defects in endoscopic anterior skull base surgery.

Methods: Thirty-six patients awaiting endoscopic anterior skull base surgery were prospectively recruited. A nasoseptal flap was used for reconstruction in all cases. Patients were assessed pre-operatively and 90 days post-operatively via the Sino-Nasal Outcome Test 20 questionnaire and visual analogue scales for nasal obstruction, pain, secretions and smell; endoscopic examination findings and mucociliary clearance times were also recorded.

Results: Sino-Nasal Outcome Test 20 questionnaire data and visual analogue scale scores for pain, smell and secretions showed no significant differences between pre- and post-operative outcomes, with visual analogue scale scores for nasal obstruction actually showing a significant improvement $(p=0.0007)$. A significant deterioration for both flap and non-flap sides was demonstrated post-operatively on endoscopic examination ( $p=0.002$ and $p=0.02$ respectively).

Conclusion: Whilst elevation of a nasoseptal flap in endoscopic surgery of the anterior skull base engendered significant clinical deterioration on examination post-operatively, quality of life outcomes showed that no such deterioration was subjectively experienced by the patient. In fact, there was significant nasal airway improvement following nasoseptal flap reconstruction.
\end{abstract}

Key words: Natural Orifice Endoscopic Surgery; Pedicled Flap; Paranasal Sinus Disease; Pituitary Neoplasms; Hypophysectomy

\section{Introduction}

Patients suffering with sellar and suprasellar lesions of the anterior skull base can present challenges for physicians. Such patients often have complex issues; in order to attain a successful outcome, these issues need to be managed with care and thought, in a multidisciplinary manner. Traditionally, the therapeutic options have included medical treatments, external approach surgery and radiotherapy. External transcranial and transfacial approaches may leave patients with cosmetic and functional morbidity. ${ }^{1}$ However, with the advent of transnasal endoscopic techniques for anterior skull base surgery, patient-related quality of life measures have revealed a perception of improved long-term outcomes. ${ }^{2,3}$
In transnasal endoscopic sellar and suprasellar surgery, performed to resect the disease in question, access is gained via the nasal cavity and paranasal sinuses. It may therefore be expected that, in the immediate post-operative period, nasal morbidity may be present. ${ }^{4}$ There are few studies that assess the degree of morbidity endured by patients after reconstruction of the skull base. ${ }^{4,5}$

Depending on the degree of the anterior skull base defect following disease removal, a pedicled flap of nasal septum mucoperiosteum and mucoperichondrium may be used to close the defect and prevent cerebrospinal fluid leak. ${ }^{6}$ The nasoseptal flap is an axial pattern flap based on the posterior septal artery, now commonly used to repair skull base defects. ${ }^{6}$ The 
nasoseptal flap is raised from the septum and swung up on its posterior pedicle to cover the surgical defect. The septum donor site takes time to heal by secondary intention before remucosalisation finally occurs.

During this healing phase, patients may potentially suffer sinonasal symptoms secondary to the nasoseptal flap elevation, including nasal obstruction, anosmia, facial pain and rhinorrhea. Furthermore, mucociliary clearance times of the nasal cavity may be altered because of the interruption to the mucosa caused by the raising of the nasoseptal flap.

This study aimed to prospectively compare the preoperative nasal symptoms with the post-operative nasal symptoms associated with the repair of the skull base defects using the nasoseptal flap, by means of the Sino-Nasal Outcome Test 20 (SNOT-20) questionnaire, visual analogue scales (VASs) for sinonasal disease symptoms, clinically based endoscopic examination and mucociliary clearance time measurement.

\section{Materials and methods}

Ethical approval was obtained from the human research ethics committee of Greenslopes Private Hospital (Brisbane, Australia). All patients provided informed consent for inclusion in the study. Patients were recruited prospectively between 2008 and 2010 in the ENT and neurosurgical departments.

\section{Inclusion criteria}

All patients scheduled for transnasal endoscopic sellar and suprasellar tumour resection and reconstruction using a nasoseptal flap were eligible for inclusion. None of the patients had any pre-operative clinical signs or symptoms of sinus disease related to the skull base lesion and had normal paranasal sinuses. Further inclusion criteria were a minimum of three months' follow up, and the ability to comply with post-operative instructions and complete outcome questionnaires.

All patients with chronic sinus disease or those who had undergone previous nasal surgery or endoscopic skull base surgery were excluded from the study. Patients requiring formal septoplasty, rather than simply posterior septectomy, were also excluded.

\section{Sinonasal outcome assessment and visual analogue scales}

Thirty-six patients were prospectively recruited. Preoperative and 90-day post-operative sinonasal outcome data and endoscopic examination findings were collected for all patients. Sinonasal outcome was assessed using the validated SNOT-20 questionnaire and VASs for nasal obstruction, nasal pain, nasal secretions and smell (scoring for the VASs ranged from 0 (no symptoms) to 10 (most severe symptoms)).

\section{Nasal endoscopy}

Pre- and post-operative nasal endoscopic examinations were all performed by the senior author, to ensure consistency. Nasal endoscopy scoring involved the use of a template, as described previously by Wallwork et al., in which the following aspects were graded: polyps $(0=$ absent or $1=$ present $)$, nasal secretions $(0=$ normal, $1=$ watery, $2=$ mucoid or $3=$ purulent $)$, swelling $(0=$ no swelling, $1=$ mild swelling or $2=$ severe swelling $)$ and mucosal colour $(0=$ pale or $1=$ red). ${ }^{7}$ A minimum score of 0 and a maximum score of 7 was obtainable.

\section{Mucociliary clearance time measurement}

Of the 36 patients in the study group, 15 were randomly selected for mucociliary clearance time measurement. However, only 12 performed both the pre-operative and 90-day post-operative mucociliary clearance time tests. A standardised process was undertaken via anterior rhinoscopy, whereby five crystals of saccharin were placed, using a plastic pipette, at the most anterior end of the inferior turbinate on the ipsilateral side to the nasoseptal flap. The time to sensation of taste, as perceived by the patient, was then recorded in minutes.

\section{Surgical technique}

The nasoseptal flap was raised as described by Hadad et al. ${ }^{6}$ The technique involved using a monopolar diathermy needle. Two parallel incisions were created, one just under the sphenoid ostium and the other along the roof of the posterior choana, so as to include the posterior septal artery in the pedicle of the flap (Figure 1). These parallel incisions were taken as laterally as possible to the sphenopalatine foramen, to allow for maximum rotation of the flap. The incisions were then extended medially along the septum superiorly and inferiorly, and joined anteriorly usually as far forward as possible (Figure 2). The vomerine rostrum was drilled back inferiorly at the pedicle region to allow the flap to be swung into position and sit flush

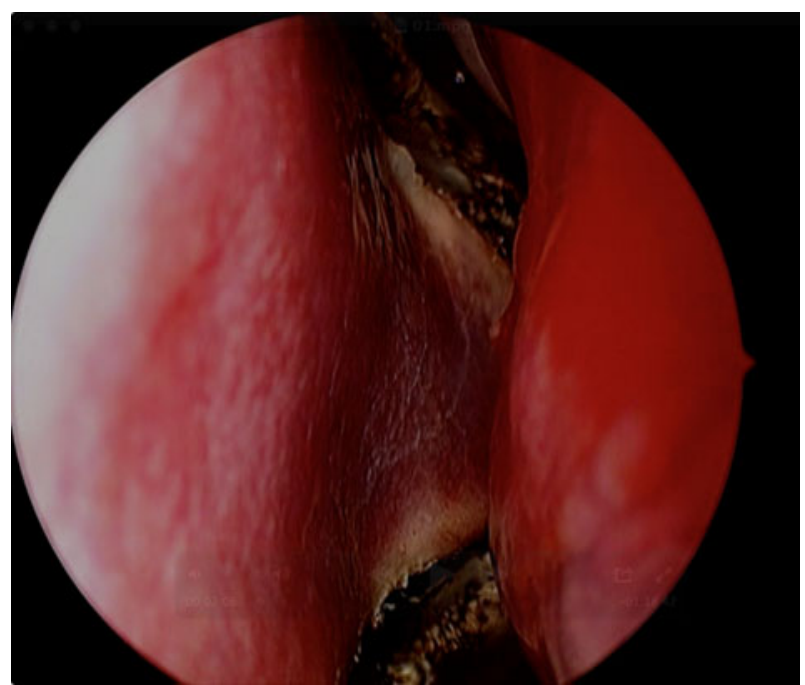

FIG. 1

Isolation of pedicle (left) 


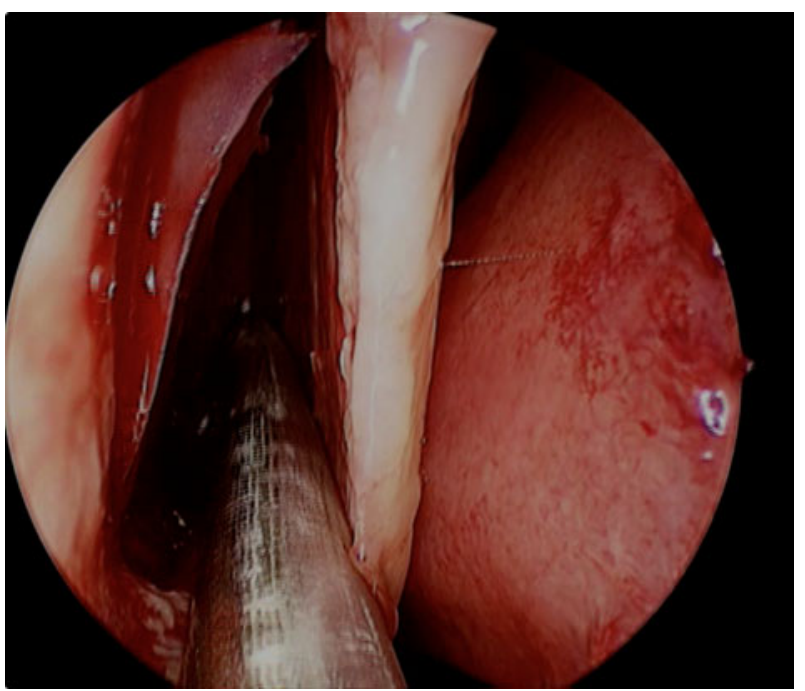

FIG. 2

Raising of nasoseptal flap at anterior septum (left)

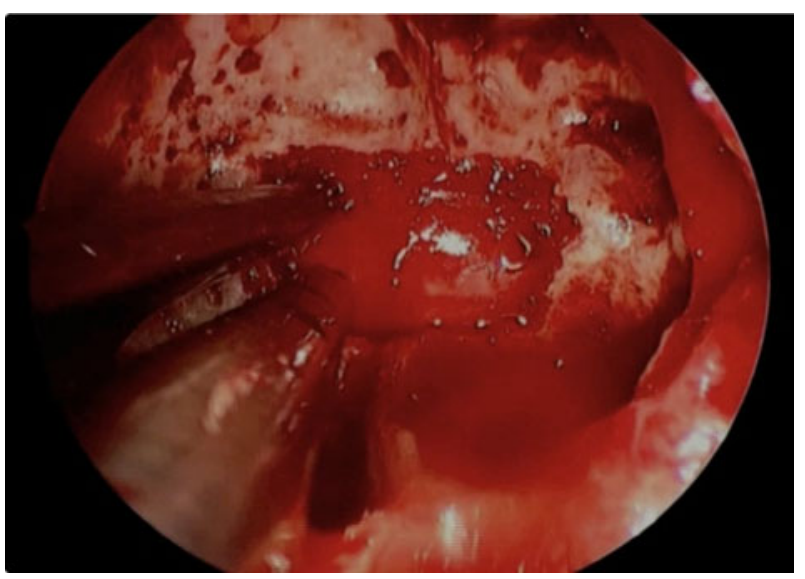

FIG. 3

Transsphenoidal skull base defect.

to the trans-sphenoidal skull base defect (Figures 3 and 4). If it was necessary for access, middle turbinates were out-fractured and not resected.

\section{Statistics}

Data analysis was performed using non-parametric statistical testing: the Wilcoxon signed rank test was used to calculate the degree and significance of correlations between pre- and post-operative data.

\section{Results}

Of the 36 patients recruited for the study, 19 had the nasoseptal flap raised from the left and 17 had it raised from the right. The senior author was the only ENT surgeon involved in the raising of the nasoseptal flaps, but three neurosurgeons were involved in the intracranial component of the surgery.

The sella and suprasellar lesions were: non-functioning macroadenoma $(n=19)$, secreting macroadenoma or

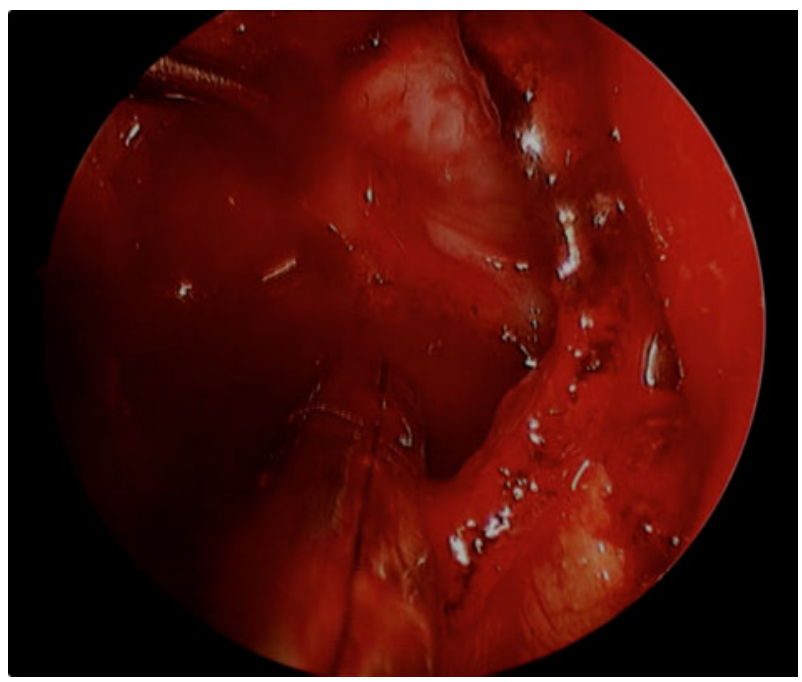

FIG. 4

Flap rotated to cover defect.

microadenoma $(n=6)$, Rathke's pouch cyst $(n=4)$, craniopharyngioma $(n=4)$, and acromegaly $(n=3)$.

Patients were assessed three months post-operatively, at their last routine ENT surgical follow-up visit. Despite the existence of literature suggesting that it may take up to six months for symptoms and quality of life scores to stabilise after endoscopic sinus surgery, three months was deemed adequate by the investigators, as patient procedures were not being performed as treatment for a primary sinonasal pathology, but rather for purposes of access only.

\section{Sino-Nasal Outcome Test 20 data}

The median pre-operative SNOT-20 questionnaire score was 1.38 (95 per cent confidence interval $(\mathrm{CI})=1.26-1.79)$ with a three-month post-operative median score of 1.65 (95 per cent $\mathrm{CI}=1.45-1.89$ ) (Figure 5). A Wilcoxon matched-pairs signed rank analysis revealed no significant difference between the preand post-operative scores $(p=0.10)$.

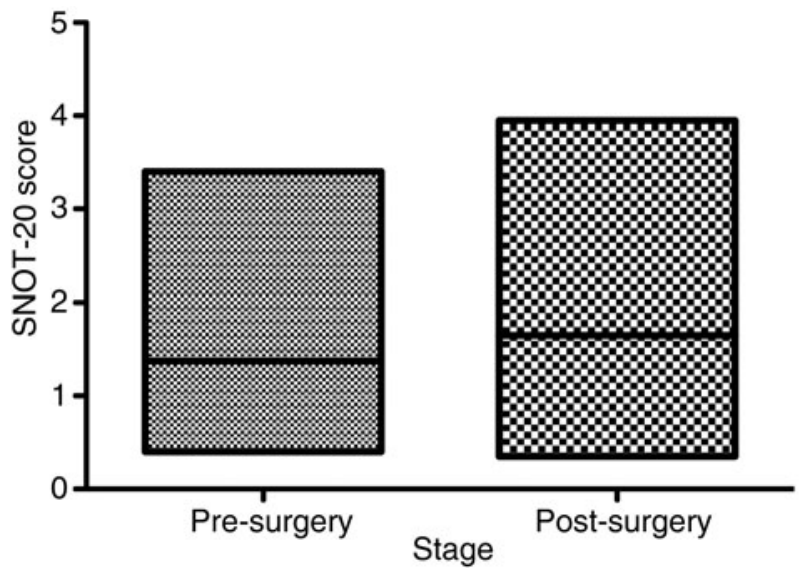

FIG. 5

Median pre- and post-operative Sino-Nasal Outcome Test 20 (SNOT-20) questionnaire scores. 


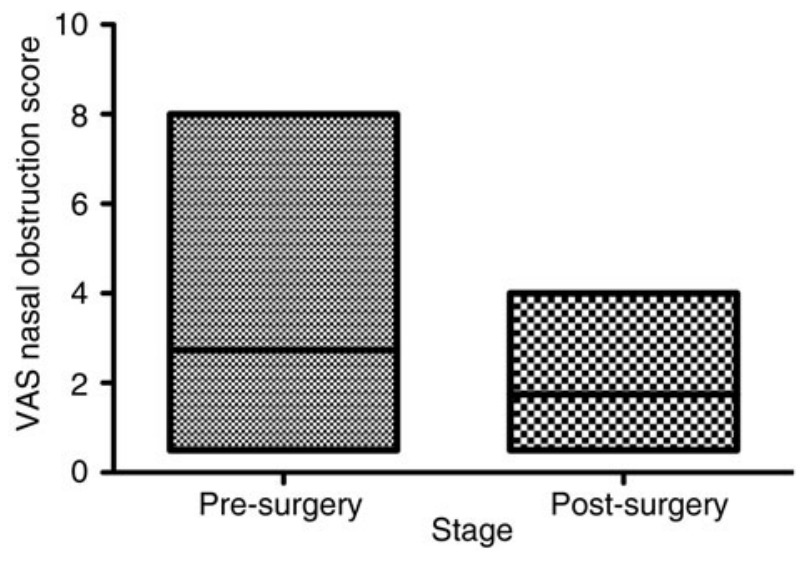

FIG. 6

Median pre- and post-operative visual analogue scale (VAS) scores for nasal obstruction.

\section{Visual analogue scale scores}

The median pre-operative VAS score for nasal obstruction was 2 (95 per cent $\mathrm{CI}=2.09-3.39)$ and the postoperative score was 1.5 (95 per cent $\mathrm{CI}=1.45-2.04$ ) (Figure 6). The mean pre- and post-operative scores were 2.74 (standard deviation $(\mathrm{SD})=1.92$ ) and 1.75 $(\mathrm{SD}=0.88)$ respectively. A Wilcoxon matchedpairs signed rank analysis revealed statistically significant nasal obstruction improvement post-operatively $(p=0.0007)$. There were no significant differences between the pre- and post-operative VAS scores for pain, secretion and smell, however $(p=0.19, \quad p=0.09$ and $p=0.37$ respectively).

\section{Nasal endoscopy findings}

The pre-operative median nasal endoscopy score on the flap side was 1 (95 per cent $\mathrm{CI}=0.78-1.5)$, with a mean score of 1.14 ( $\mathrm{SD}=1.05)$. Post-operatively, the ipsilateral median nasal endoscopy score was 2 (95 per cent $\mathrm{CI}=1.66-2.34)$, with a mean score of 2 $(\mathrm{SD}=1.01)$. A Wilcoxon matched-pairs signed rank analysis showed a significant difference between the pre- and post-operative nasal endoscopy findings $(p=0.002)$. A significant difference was also found on the contralateral or non-nasoseptal flap side $(p=0.02)$.

\section{Mucociliary clearance times}

Fifteen patients in our study underwent pre-operative mucociliary clearance time testing; however, only 12 of those patients performed the 3-month post-operative mucociliary clearance time test. The median pre-operative mucociliary clearance time was 13.5 minutes (i.e. 13 minutes and 30 seconds) (95 per cent $\mathrm{CI}=$ 10.33-16.5) (Figures 7 and 8). The median post-operative time was 10 minutes $(95$ per cent $\mathrm{CI}=$ 8.71-11.45). The mean pre- and post-operative times were 13.42 minutes $(\mathrm{SD}=5.58)$ and 10.08 minutes $(\mathrm{SD}=2.16)$ respectively. A Wilcoxon matched-pairs signed rank analysis revealed no significant difference between the pre- and post-operative mucociliary

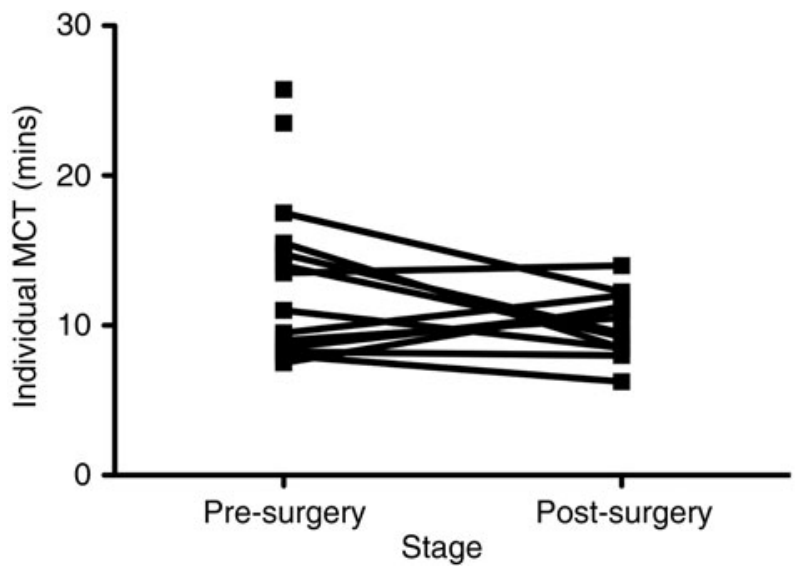

FIG. 7

Individual patient mucociliary clearance times (MCT).

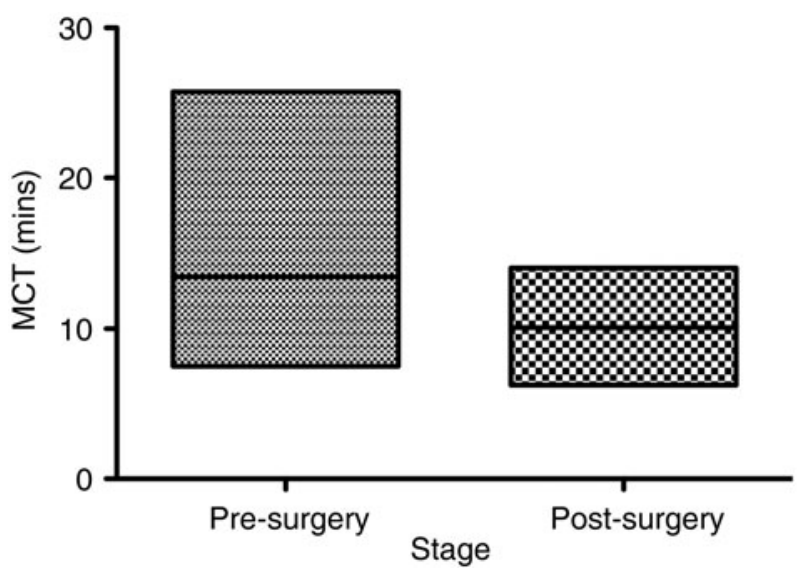

FIG. 8

Median mucociliary clearance times (MCT).

clearance times $(p=0.29)$. However, Spearman approximation found that this pairing was not significantly effective and, hence, the non-significance of the Wilcoxon signed rank analysis cannot be relied upon.

\section{Discussion}

Traditional microscopic techniques for transsphenoidal pituitary surgery used to be the 'gold standard' for management of pituitary tumours. However, endoscopic skull base techniques offer much greater local visualisation, improved surgical outcome and reduced morbidity. ${ }^{8}$ Endoscopic techniques have further evolved to include nasoseptal flap reconstruction of the skull base. ${ }^{6}$ Access and reconstructive elements form only components of such procedures. As such, an inherent difficulty exists in distinguishing the contribution that these factors make to the patient morbidity profile from the contribution of other procedural elements, namely the complex nature of such surgery and the repeated use of neurosurgical instruments in the nasal cavity. 
de Almeida et al. performed a study to assess nasal morbidity following endoscopic skull base surgery, and found that it was related to the complexity of the surgical operation. ${ }^{4}$ Nasal crusting was found in 98 per cent of patients at one month, in addition to nasal discharge, smell and taste disturbance. The crusting reduced over time, with only 50 per cent of all patients still experiencing crusting at three months. More importantly, the authors failed to show a difference in the duration of nasal crusting when comparing patients who had undergone nasoseptal flap reconstruction against those who had not.

The nasoseptal flap has demonstrated efficacy as a reconstructive tool; however, no previous study has primarily assessed the nasal morbidity associated with its use. de Almeida et al. assessed nasal morbidity across different surgical approaches to the skull base for a variety of disease processes. In contrast, in our study, all 36 patients underwent endoscopic transsphenoidal procedures for sellar or suprasellar lesions, and all with nasoseptal flap reconstruction. Interestingly, with these strict inclusion criteria, we clinically observed significant differences in nasal endoscopic examination scores on both the flap and non-flap sides at three months post-operatively $(p=0.002)$. Like de Almeida, these findings suggest clinical evidence of continued nasal morbidity, which is to be expected up to three months post-operatively.

Nevertheless, using the disease-specific instrument SNOT-20, we found that patients did not report a significant reduction in their quality of life at three months post-operatively, despite the significant differences observed for nasal endoscopy findings (Figure 5). In keeping with this, there were no significant differences in VAS scores for pain, smell or nasal secretion for our patient cohort. Furthermore, we found significant patient-reported nasal obstruction improvement at three months compared with preoperative VAS scores $(p=0.0007)$ (Figure 6). Again, patient-reported experiences were not in line with our observed clinical examination findings (Figure 9), which suggested significantly worse nasal endoscopic examination findings post-operatively $(p=0.002)$.

Ryan et al., amongst others, report a poor correlation between patient-related outcome measures and clinical examination findings in patients with skull base disease. ${ }^{9,10}$

Further work must be undertaken to assess our preliminary findings of significant patient-reported nasal airway improvements (in patients with a nasoseptal flap in whom the donor site was exposed to allow it to remucosalise over time). This effect may be the result of an increase in the nasal volume of Cottle's area around the septal wall anteriorly, or because of rotation of the erectile septal flap containing tissue posteriorly. Other features of the procedure, such as middle turbinate out-fracturing and posterior septectomy, may also contribute to the patient-perceived change in nasal obstruction (Figure 10). However, Caicedo-Granados

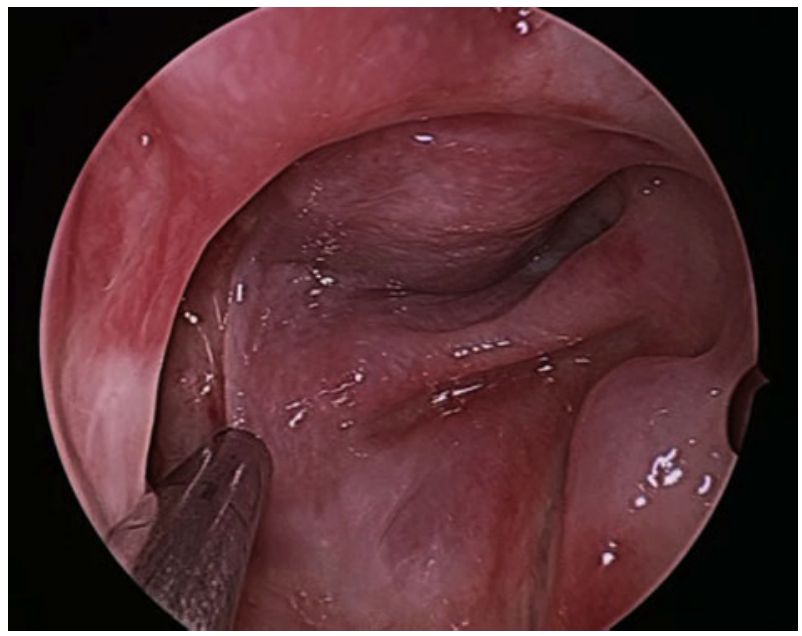

FIG. 9

Flap healed post-operatively.

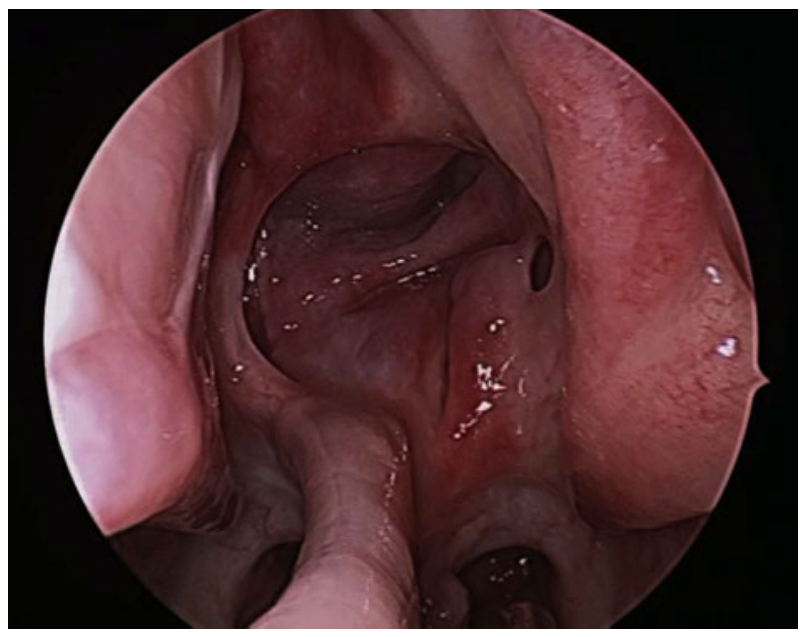

FIG. 10

Healthy sinonasal cavity post nasoseptal flap reconstruction.

et al. report a reverse septal rotation flap used to cover the donor site, and document patient-reported improvements in nasal crusting, airway and quality of life, with a reduction in nasal toilets. ${ }^{11}$ A randomised study assessing nasal airway with and without the reverse rotation flap may well enlighten us further on this subject.

Pant et al. recently performed a study to assess the quality of life in patients undergoing endoscopic skull base surgery. ${ }^{5}$ They acknowledged the lack of disease-specific quality of life outcome measures in patients with skull base tumours. They used a multidimensional outcome measure as well as the SNOT20 questionnaire. ${ }^{5,12}$ Their results revealed a significant improvement in the short-term quality of life scores for patients who did not undergo nasoseptal flap reconstruction compared with those that did. However, overall, they suggest that there was a temporary reduction in the short-term quality of life following endoscopic skull base surgery, which was linked to nasal 
morbidity. The SNOT-20 results also indicated that their patients found smell or taste, nasal obstruction, postnasal discharge, waking up at night, and lack of sleep to be the most important factors affecting their quality of life. ${ }^{5}$

To our knowledge, no previous studies have assessed mucociliary clearance times in endoscopic skull base surgery patients. In our study, the results for the 12 patients that completed pre- and post-operative mucociliary clearance time testing revealed a median change from 13.5 to 10 minutes. This change was not significantly different. However, our analysis suggested that, most likely because of the lack of patient numbers, any statistical analysis would not obtain sufficient power for statistical calculation. Nonetheless, the results suggest there may not be a difference in pre- and post-operative mucociliary clearance times and are certainly encouraging (Figures 7 and 8). Further work may be needed in this area to assess the impact of nasoseptal flap reconstruction on nasal physiology following endoscopic skull base surgery.

- Endonasal surgery of the anterior skull base has improved patient morbidity compared with traditional 'open' approaches

- The nasoseptal pedicled flap has proven efficacy as a safe reconstructive option for skull base defects post-excision

- The nasoseptal flap is well-tolerated by patients, with no significant increase in adverse sinonasal symptoms

- Patients actually report significant nasal obstruction improvement following nasoseptal flap reconstruction in anterior skull base surgery

The authors acknowledge that the use of a control group, comprising patients who did not undergo nasoseptal flap reconstruction, may have added to this trial. However, for the purposes of this study, each patient acts as their own control with the collection of pre-and post-operative results; even a double-blinded, randomised, controlled trial is not without its limitations in respect to this particular clinical question. The authors further acknowledge that improvements could be made to reduce confounding within the study. For instance, the number of patients that participate in the mucociliary clearance time tests could be increased, an independent specialist could be asked to examine the nasal cavities pre- and post-operatively, and a disease-specific outcome measure for endoscopic assessment and approaches could be utilised. However, no outcome measures currently exist for endoscopic skull base surgery, and the overriding message is clear: patients have limited nasal morbidity.

\section{Conclusion}

Whilst our results suggest significant clinical deterioration on nasal examination, patient-reported quality of life outcomes suggest no such perceived deterioration at three months post-operatively. In fact, the findings suggest significant subjective airway improvement following nasoseptal flap reconstruction. With much improved recovery times and global improvement in quality of life, short-term nasal morbidity may be a small price to pay for an endoscopic approach to, and nasoseptal flap reconstruction of, the anterior skull base.

\section{References}

1 Catalano PJ, Hecht CS, Biller HF, Lawson W, Post KD, Sachdev V et al. Craniofacial resection. An analysis of 73 cases. Arch Otolaryngol Head Neck Surg 1994;120:1203-8

2 Amit M, Abergel A, Fliss DM, Gil Z. The clinical importance of quality-of-life scores in patients with skull base tumors: a metaanalysis and review of the literature. Curr Oncol Rep 2012;14: $175-81$

3 McCoul ED, Anand VK, Bedrosian JC, Schwartz TH. Endoscopic skull base surgery and its impact on sinonasalrelated quality of life. Int Forum Allergy Rhinol 2012;2:174-81

4 de Almeida JR, Snyderman CH, Gardner PA, Carrau RL, Vescan AD. Nasal morbidity following endoscopic skull base surgery: a prospective cohort study. Head Neck 2011;33:547-51

5 Pant H, Bhatki AM, Snyderman CH, Vescan AD, Carrau RL, Gardner P et al. Quality of life following endonasal skull base surgery. Skull Base 2010;20:35-40

6 Hadad G, Bassagasteguy L, Carrau RL, Mataza JC, Kassam A, Snyderman $\mathrm{CH}$ et al. A novel reconstructive technique after endoscopic expanded endonasal approaches: vascular pedicle nasoseptal flap. Laryngoscope 2006;116:1882-6

7 Wallwork B, Coman W, Mackay-Sim A, Greiff L, Cervin A. A double-blind, randomized, placebo-controlled trial of macrolide in the treatment of chronic rhinosinusitis. Laryngoscope 2006; 116:189-93

8 Stammberger H, Anderhuber W, Walch C, Papaefthymiou G. Possibilities and limitations of endoscopic management of nasal and paranasal sinus malignancies. Acta Otorhinolaryngol Belg 1999;53:199-205

9 Ryan WR, Ramachandra T, Hwang PH. Correlations between symptoms, nasal endoscopy, and in-office computed tomography in post-surgical chronic rhinosinusitis patients. Laryngoscope 2011;121:674-8

10 Gil Z, Abergel A, Spektor S, Khafif A, Fliss DM. Patient, caregiver, and surgeon perceptions of quality of life following anterior skull base surgery. Arch Otolaryngol Head Neck Surg 2004; 130:1276-8

11 Caicedo-Granados E, Carrau R, Snyderman CH, Prevedello D, Fernandez-Miranda J, Gardner P et al. Reverse rotation flap for reconstruction of donor site after vascular pedicled nasoseptal flap in skull base surgery. Laryngoscope 2010;120:1550-2

12 Gil Z, Abergel A, Spektor S, Cohen JT, Khafif A, Shabtai E et al. Quality of life following surgery for anterior skull base tumours. Arch Otolaryngol Head Neck Surg 2003;129:1303-9

Address for correspondence:

Dr Martin Hanson,

Department of Otolaryngology,

Head and Neck Surgery,

Princess Alexandra Hospital,

Woolloongabba,

Queensland 4102, Australia

E-mail: martin.hanson1@uqconnect.edu.au

Dr M Hanson takes responsibility for the integrity

of the content of the paper

Competing interests: None declared 